\title{
CYP1A1, GSTM1 and GSTT1 polymorphisms and oral cancer - Correlation with patients' status at 2-year, age of onset, nodal status, tumor size and stage
}

Altered expression of xenobiotic enzymes such as CYP1A1,GSTM1 and GSTT1 has been reported in some malignant tumors including oral cancer. However, the correlation between these enzymes and clinicopathologic parameters has not been well documented. The aim of this study was to investigate the associations between CYP1A1, GSTM1 and GSTT1 polymorphisms with patients' status at 2-year, age of onset, nodal status, tumor size and disease stage. A total of 195 oral cancer patients were included in this study. Peripheral blood was obtained from consented individuals and CYP1A1, GSTM1 and GSTT1 genotypes were determined using PCR and restriction enzyme digestion. Chi-square test and simple logistic regression yielding odds ratio $(\mathrm{OR})$ was employed for comparison of all parameters except for age of onset where t-test was used. Patients with GSTM1/GSTT1 polymorphism were associated with almost triple increased risk of mortality at 2-year after the first being diagnosed (OR 2.79, 95\% Cl 1.22-6.36). Mean age of onset was higher in those with GSTT1 null (60.82 \pm 10.61 years) and GSTM1/GSTT1 polymorphism genotype (59.48 \pm 11.30 years) compared to lower mean age of onset for those with GSTT1 non-null (58.15 \pm 11.97 years) and GSTM1/GSTT1 wild-type genotype and these observations were not significant. There was also no significant difference between CYP1A1 and GSTM1 polymorphisms and age of onset. Positive nodes are associated with high CYP1A1 polymorphism, GSTM1 null and GSTM1/GSTT1 polymorphism $(59.7 \%, 56.5 \%$

and $72.6 \%$, respectively) and low GSTT1 null (29.0\%). For tumor size of more than $2 \mathrm{~cm}$, there was higher percentage of GSTT1 null genotype (92.7\%) as compared to those with tumor size of less than $2 \mathrm{~cm}(p=0.035)$. Polymorphisms of all three genes were higher in late stage compared to early stage disease. However, statistically, these observations were not significant. In conclusion, no association was observed between CYP1A1 and GSTM1 polymorphisms and all clinicopathologic parameters studied. This study also showed that for GSTM1/GSTT1 and GSTT1 polymorphisms, association was seen with patients' status at 2-year and tumor size respectively, indicating that the GST genotypes may be important indicators for the patients' status and tumor size in oral cancer. 


\begin{tabular}{|c|l|}
\hline Author & $\begin{array}{l}\text { Abraham, M. T. ;Karen-Ng, L. P. ;Ghani, W. M. N. ;Zain, R. B. ;Raja- } \\
\text { Latifah, R. J. ;Cheong, S. C. }\end{array}$ \\
\hline Source & Oral Oncology \\
\hline ISSN & $1368-8375$ \\
\hline DOI & $10.1016 /$ j.oos.2009.06.582 \\
\hline Page & $219-220$ \\
\hline Year & 2009 \\
\hline
\end{tabular}

\section{Please Cite As}

Abraham MT, Karen-Ng LP, Ghani WMN, Zain RB, Raja-Latifah RJ, Cheong SC. CYP1A1, GSTM1 and GSTT1 polymorphisms and oral cancer - Correlation with patients' status at 2-year, age of onset, nodal status, tumor size and stage. Oral Oncology. [Meeting Abstract]. 2009 Jul:219-20.

URL

- http://apps.webofknowledge.com/full record.do?product=WOS\&search mode= MarkedList\&qid=4\&SID=P2NFmdeflc@cFHgm9J2\&page=1\&doc=1\&colName= WOS

- http://www.sciencedirect.com/science/article/pii/S1744789509005835 